\title{
Adherence of Subjects With Cystic Fibrosis to Their Home Program: A Systematic Review
}

\author{
Ruairi O’Donohoe and Brona M Fullen MSc PhD
}

\begin{abstract}
BACKGROUND: The management of cystic fibrosis (CF) includes adherence to a home management program (airway clearance, medication, nutritional advice, and exercise). This has led to an increase in life expectancy, although the benefits depend greatly on a patient's level of adherence to daily treatments at home. To date, no systematic review has established adherence rates to all World Health Organization guidelines in the home setting; hence, this review was undertaken. METHODS: The review comprised 3 phases. A methodological assessment of databases (Embase, CINAHL, PsychINFO, PEDro, PubMed, Cochrane Central Register of Controlled Trials) identified potentially relevant papers. These papers were screened for inclusion criteria by 2 independent reviewers, data were extracted, and the internal validity was rated using a valid and reliable scale. Results were categorized into 4 themes: medication, nutrition, airway clearance techniques, and exercise. RESULTS: The search generated a total of 26 papers, 24 of which were rated as being poor quality. Adherence to a treatment program for CF patients is generally low (from $22 \%$ for nutritional guidelines to $130 \%$ for oral antibiotics), and it varies greatly depending on the type of treatment and the method of assessment employed (objective tool vs self-reported questionnaires). CONCLUSIONS: Consensus on how to measure adherence is lacking, and the quality of studies addressing adherence in this population is generally poor. Overall, studies using self-reported measures yielded higher adherence scores than those that used objective measures, suggesting that current efforts to improve methods of adherence are appropriate. The prevalence of non-adherence remains unclear due to these limitations. Key words: cystic fibrosis; adherence; home treatment program; systematic review. [Respir Care 2014;59(11):1731-1746. (C) 2014 Daedalus Enterprises]
\end{abstract}

\section{Introduction}

The World Health Organization (WHO) guidelines for the management of cystic fibrosis (CF) include airway clearance, medication (antibiotics, pancreatic enzyme replacement therapy), nutrition (high-calorie diet, nutritional supplements), and exercise. ${ }^{1}$ These strategies have led to an increased life expectancy, which has increased from 28

The authors are affiliated with the School of Public Health, Physiotherapy and Population Science, Health Science Centre, University College Dublin, Dublin, Ireland.

The authors have disclosed no conflicts of interest.

Correspondence: Brona M Fullen MSc PhD, School of Public Health, Physiotherapy and Population Science, Health Science Centre, University College Dublin, Dublin, Ireland. E-mail: brona.fullen@ucd.ie.

DOI: $10.4187 /$ respcare. 02990 to $37 \mathrm{y}$ in the past 2 decades in the United States. ${ }^{2}$ However, the benefits of these new treatments depend greatly on the patients' level of adherence to daily treatments at home. Treatments can be burdensome, time-consuming, and costly, and given the long-term and arduous nature of the regimes, poor adherence is commonly reported. ${ }^{3}$ The daily regimen can include ingesting as many as $40-50$ pills, inhalation treatments requiring up to $2 \mathrm{~h}$, and 2-3 airway clearance sessions lasting $30 \mathrm{~min}$ each. In addition, patients are encouraged to exercise and eat several highcalorie, high-fat meals each day. ${ }^{4}$ The average number of treatments per day has been calculated as 7 , taking an average of 108 min. ${ }^{5}$ In addition, the maintenance of equipment and preparation of medications (and overcoming administrative barriers to maintaining access to medications) add to the time burden. ${ }^{6}$ Individual studies have reported mainly on adherence rates to some of the recommended treatments, for example, medication and chest physical therapy. Adherence rates have been reported to be $67 \%$ for 
oral antibiotics, $31-53 \%$ for inhaled antibiotics, ${ }^{7} 33 \%$ for dornase alfa, and $40-70 \%$ for chest physical therapy. $3,8-12$

Although adherence levels for subcategories of 2 of the 4 WHO recommendations have been the subject of systematic reviews (adherence to antibiotics only, ${ }^{13}$ airway clearance techniques ${ }^{14}$ ), the inclusion of randomized controlled trials may not reflect participants' level of adherence to their independent home treatment regimes due to the nature of frequent monitoring as part of being involved in a trial. Hence, this review was undertaken to include all 4 WHO recommendations (nutrition, medication, exercise, pancreatic enzyme replacement therapy) in non-interventional studies.

Poor adherence to treatment results in increased morbidity and mortality, a reduction in quality of life, and increased health-care utilization. ${ }^{15-17}$ Briesacher et al ${ }^{18}$ demonstrated both a $60 \%$ decrease in the risk of hospitalization for people who were adherent to their treatment regimen and a relationship between low usage of chronic medications and increased exacerbations requiring intravenous therapy. Improving patient outcomes rests, in part, on increasing adherence to chronic therapies, and identifying reasons for non-adherence to treatment is important.

Thus, due to the multiple facets of CF treatment (exercise, nutritional advice, airway clearance techniques, and medication) as recommended by the $\mathrm{WHO}^{19}$ and the importance of determining adherence in observational rather than formal research settings, this review was undertaken.

\section{Methods}

\section{Overview}

The review comprised 3 phases. Phase 1 involved a systematic search of the literature using devised criteria and a search strategy of key words. Phase 2 involved the initial screening of appropriate abstracts and subsequently entire papers by 2 independent reviewers (RO'D and Ms Anastasia Kitova [School of Public Health, Physiotherapy and Population Science, University College Dublin, Dublin, Ireland]). Phase 3 involved classifying the internal validity of the included papers and grading the strength of the evidence using the Effective Public Health Practice Project quality assessment tool for quantitative studies. ${ }^{20}$

\section{Phase 1: Search Strategy}

Electronic searches of PubMed, CINAHL, Embase, PEDro, and the Cochrane Central Register of Controlled Trials were carried out from January 1992 to October 2012 on October 30, 2012. A battery of key words was developed using the WHO guidelines for the diagnosis and management of cystic fibrosis ${ }^{19}$ to identify the various

\section{QUICK LOOK}

\section{Current knowledge}

Successful treatment of cystic fibrosis requires home management, including nutrition, airway clearance, exercise, and pharmacotherapy. Good adherence to home management is associated with improved quality of life.

\section{What this paper contributes to our knowledge}

A systematic review of the literature suggests that adherence is quite variable and that studies are commonly of poor quality. Self-reported adherence rates are routinely higher than adherence rates measured by objective measures. These data suggest that continued efforts to improve adherence are warranted.

treatment strategies used for CF. Key terms included cystic fibrosis AND adherence AND treatment plan AND exercise OR airway clearance techniques OR diet OR medication. Appropriate synonyms of each search term were used in each search string, along with suitable MeSH terms found in the PubMed database. The complete search strategy is summarized in Table 1. The same search strategy was used for PubMed (Medline), CINAHL (EBSCOhost), Embase (Elsevier), and Cochrane. The search terms used in PEDro included cystic fibrosis AND adherence.

The inclusion criteria were studies involving patients with CF, studies relating to patients' home treatment, studies focusing on adherence as the primary or secondary measure, and studies published from 1992 to 2012. Exclusion criteria were studies published before 1992, intervention studies, review papers, studies conducted in a hospital setting, studies related to exacerbations, and studies that appeared only as abstracts in supplements or poster sessions. Hand searches were also conducted on the bibliographies of identified articles for relevant papers.

\section{Phase 2: Screening Process and Data Extraction}

Potentially relevant articles were identified from the titles, abstracts, and key words of the references retrieved by the literature search, and they were scrutinized by 2 researchers (RO'D and AK) for inclusion/exclusion criteria. Duplicates were removed, and the entire papers of accepted abstracts were retrieved and independently scrutinized by the 2 researchers using a detailed pro forma developed to capture and subsequently categorize relevant information from the included papers (participants' demographic data, study methodology, results). Accepted papers were re-categorized into themes: medication, airway clearance techniques, nutrition, and exercise. 
Table 1. Database Search Strategy

\begin{tabular}{l}
\hline Date of Search \\
Years \\
Search Strings \\
(1) Patient group \\
(2) Focus of study \\
(3) Airway clearance strategies
\end{tabular}

(4) Exercise

(5) Medication therapy

(6) Dietary recommendations

(7) General treatment

(8) Regime

Combination of terms

Final search

Limitations
October 28, 2012

No restriction

Cystic fibrosis [MeSH] OR cystic fibrosis OR CF OR mucoviscidosis

Medication adherence [MeSH] OR guideline adherence [MeSH] OR patient compliance [MeSH] OR adherence OR compliance OR conformability OR obedience OR accordance

Chest physiotherapy OR CPT OR conventional chest physiotherapy OR CCPT OR postural drainage OR percussion [MeSH] OR chest percussion OR clapping OR vibration OR high frequency chest wall oscillations OR chest wall oscillation [MeSH] OR HFCWO OR coughing exercises OR coughing OR cough [MeSH] OR ACBT OR active cycle of breathing technique OR breathing control OR BC OR thoracic expansion exercises OR thoracic expansion OR TEE OR forced expiratory technique OR FET OR AD OR autogenic drainage OR oscillating positive pressure device OR flutter OR PEP OR PEP mask OR PEP device OR positive expiratory pressure device OR positive expiratory pressure mask OR positive-pressure respiration $[\mathrm{MeSH}] \mathrm{OR}$ high pressure positive expiratory pressure OR high pressure PEP OR HPEP

Exercise OR exercise [MeSH] OR exercise therapy [MeSH] OR exercise movement techniques OR resistance training [MeSH] OR muscle stretching exercises [MeSH] OR breathing exercises [MeSH] OR train OR training OR work out OR working out OR activity OR physical activity OR motor activity [MeSH] OR PA OR strengthening OR stretching OR sport OR sports [MeSH] OR aerobic OR anaerobic

Inhalation therapy OR inhaler OR nebulizers and vaporizers [MeSH] OR metered dose inhalers [MeSH] OR dry powder inhalers [MeSH] OR steroids [MeSH] OR steroids OR bronchodilator agents [Pharmacological Action] OR bronchodilator agents [MeSH] OR bronchodilator OR anti-bacterial agent [MeSH] OR anti-bacterial agents [Pharmacological Action] OR antibiotic prophylaxis [MeSH] OR saline solution, hypertonic [MeSH] OR hypertonic saline OR expectorants [MeSH] OR expectorants [Pharmacological Action] OR mucolytic OR DNase OR amiloride OR UTP OR uridine triphosphate OR pancreatic enzymes

Nutrition OR diet [MeSH] OR diet therapy [MeSH] OR diet therapy [Subheading] OR diet OR vitamins [MeSH] OR vitamins [Pharmacological Action] OR supplements OR dietary supplements $[\mathrm{MeSH}]$ OR vitamin A OR vitamin K OR vitamin D OR vitamin E

Therapeutics [MeSH] OR therapy [Subheading] OR clinical protocols [MeSH] OR therapies, investigational $[\mathrm{MeSH}] \mathrm{OR}$ placebos $[\mathrm{MeSH}] \mathrm{OR}$ treatment $\mathrm{OR}$ care OR medical care OR nondirective therapy [MeSH] OR drug therapy, computer-assisted [MeSH] OR enzyme therapy $[\mathrm{MeSH}]$ OR physical therapy modalities $[\mathrm{MeSH}]$ OR recreation therapy [MeSH] OR gene therapy $[\mathrm{MeSH}]$ OR respiratory therapy [MeSH] OR enzyme replacement therapy $[\mathrm{MeSH}] \mathrm{OR}$ medication therapy management $[\mathrm{MeSH}] \mathrm{OR}$ drug therapy, combination [MeSH] OR oxygen inhalation therapy [MeSH] OR nutrition therapy [MeSH] OR exercise therapy [MeSH] OR drug therapy [MeSH] OR relaxation therapy [MeSH] OR drug therapy [Subheading] OR individualized medicine [MeSH] OR diet therapy [Subheading] OR medicine [MeSH] OR evidence-based medicine [MeSH] OR pulmonary medicine $[\mathrm{MeSH}]$ OR infectious disease medicine $[\mathrm{MeSH}] \mathrm{OR}$ preventive medicine $[\mathrm{MeSH}]$ OR physical medicine [MeSH] OR medication therapy management [MeSH] OR medication OR management

Health plan implementation [MeSH] OR plan OR program OR programme OR scheme OR regimen OR regime OR strategy OR method OR procedure OR early intervention (education) $[\mathrm{MeSH}]$ OR clinical protocols $[\mathrm{MeSH}]$ OR methods [MeSH] OR methods [Subheading]

$9=1$ AND 2 (CF and adherence)

$10=3$ OR 4 OR 5 OR 6 (all specific treatment types)

$11=7$ AND 8 (treatment plan)

$12=10$ AND 11 (all treatments encompassed)

$13=9$ AND 12

CF AND adherence AND all treatments

Humans only, English only 


\section{Adherence to Cystic Fibrosis Programs}

\section{Phase 3: Internal Validity}

An assessment of quality was carried out on each remaining paper using the Effective Public Health Practice Project quality assessment tool for quantitative studies. ${ }^{20}$ It comprises 8 components (selection bias, study design, confounders, blinding, data collection methods, withdrawal and dropouts, intervention integrity, analyses) that are individually scored, and an overall rating of the internal validity is assigned: strong, moderate, or weak. This tool has been deemed appropriate for assessing the quality of non-randomized trials as well as randomized controlled trials. $^{21}$

\section{Results}

In total, 26 papers of various methodologies were included in the review: cross-sectional studies $(n=13)$ and longitudinal design $(n=13)$. The search strategy is reported in Table 1, and included studies, study designs, results, and internal validity ratings are summarized in Tables $2-5$ and Figure 1.

\section{Characteristics of Included Studies}

Two of the 26 papers were classified as being of moderate methodological quality, 24 were of low quality, and no studies were classified as being of high quality. The review included 2,967 participants. The age range varied from 3 to $28 \mathrm{y}$, and study duration ranged from $3 \mathrm{~d}$ to $1 \mathrm{y}$. Different methods were used to determine adherence rates (eg, electronic diaries, pharmacy refills, and health insurance patient, parent caregiver, and doctor questionnaires, interviews, and telephone surveys). Questionnaires included disease-specific questionnaires (Manchester cystic fibrosis questionnaire, treatment adherence rating scale) and adherence questionnaires (disease management interview-cystic fibrosis, medical and nonmedical compliance questionnaire, confidential cystic fibrosis management profile). The inconsistency in the measurement of adherence across studies makes comparison difficult, although overall objective measures of adherence scored lower than the self-reported questionnaires. The treatment themes are discussed in detail below.

\section{Medication}

Twenty papers assessed participants' adherence to medication (male, $n=916$; female, $n=855$; unspecified, $n=69)$. 3,10,11,20,22-25,27-29,31-39 Eighteen papers were of low quality, and 2 were of moderate quality. The categories of medications are summarized below (Table 3 ).
Nebulized Antibiotics. Seven papers assessed adherence with nebulized antibiotics in 1,093 participants (male, $n=565$; female, $n=528$ ). All papers were of low quality. Some studies used multiple methods of assessment. Two studies used electronic monitoring via an adaptive aerosol delivery device, ${ }^{33,34} 3$ used participant questionnaires, ${ }^{3,25,31}$ 2 used parent questionnaires, ${ }^{3,23}$ one used daily telephone diaries, ${ }^{3}$ one used pharmacy refill data, ${ }^{35}$ and one used data from a research database of health-care claims ${ }^{20}$ to assess adherence. Mean overall percentage of adherence ranged from $36.1 \pm 35.6 \%$ by daily telephone diary ${ }^{3}$ to $85.0 \pm 33.7 \%$ by patient questionnaire. ${ }^{3}$

Antibiotics. Two studies assessed adherence to antibiotics (not focusing on specific medication/delivery) in 127 participants (male, $n=68$; female, $n=59$ ). Both studies were of low quality. One study used a participant questionnaire ${ }^{27}$; the other used a parent questionnaire. ${ }^{29}$ Mean adherence ranged from $76 \pm 28.8 \%{ }^{37}$ to $87.5 \pm 19.5 \% .{ }^{10}$

Two studies assessed adherence to oral antibiotics in 148 participants (male, $n=68$; female, $n=80$ ). Both studies were of low quality. One used pharmacy refill data, ${ }^{35}$ whereas the other used both participant and parent questionnaires. ${ }^{27}$ The mean adherence ranged from $76 \%$ by pharmacy refill ${ }^{35}$ to $130 \%$ by patient questionnaire. ${ }^{31}$

Bronchodilators. One low-quality study assessed adherence to nebulized bronchodilators in 53 participants (male, $n=22$; female, $n=31$ ) using parent and participant questionnaires. Adherence was recorded at $60 \%$ and $61 \%$, respectively. ${ }^{31}$

Undifferentiated Inhalational Therapy. Five studies assessed adherence to nebulizer therapy (not focusing on specific medication) in 192 participants (male, $n=93$; female, $n=72$; unspecified, $n=27$ ). All studies were of low quality. Four studies used participant questionnaires, 3,23,27,32 2 used clinician questionnaires, ${ }^{27,32} 2$ used parent questionnaires, ${ }^{3,27}$ one used electronic monitoring, ${ }^{32}$ and one used pharmacy refill data and daily telephone diaries. ${ }^{3}$ Adherence ranged from a median (interquartile range) of $36 \%(5-85.5 \%)$ by electronic measurement ${ }^{32}$ to $93.5 \%$ by parent report. ${ }^{28}$

Hypertonic Saline. One low-quality study assessed adherence to hypertonic saline nebulizers in 95 participants (male, $n=46$; female, $n=49$ ) using pharmacy refill data. Median (interquartile range) adherence was $49 \%$ (0$85 \%) .35$

One low-quality study reported a combined result for airway clearance techniques and nebulizers in 95 participants (male, $n=50$; female, $n=46$ ) using mother, father, and participant questionnaires. Mean adherence scores were 


\section{Adherence to Cystic Fibrosis Programs}

Table 2. Adherence to Airway Clearance Techniques

\begin{tabular}{|c|c|c|c|c|c|c|}
\hline Authors & Participants & Treatment Category & Study Duration & Measurement of Adherence & Results & Q \\
\hline Eddy et al22 & $\begin{array}{l}n=41(20 \text { males, } \\
\quad 21 \text { females }) \\
\text { Age } 6.7 \pm 2.67 \mathrm{y} \\
\text { Range } 3-11 \mathrm{y}\end{array}$ & CPT (not specified) & $\begin{array}{l}\mathrm{C} / \mathrm{S} \\
\text { General } \\
\text { adherence }\end{array}$ & $\begin{array}{l}\text { Questionnaire: } \\
\text { (1) Parent-reported adherence } \\
\text { to recommendations } \\
\text { (2) Clinician-reported: } \\
\text { Likert scale, } 1(\geq 95 \% \text { of } \\
\text { time) to } 5 \text { ( } \leq 5 \% \text { of time) }\end{array}$ & $\begin{array}{l}n, \text { mean } \pm \mathrm{SD} \\
\% \text { of total } n \text { who } \\
\text { scored } 1 \\
\text { (1) Parent-reported: } \\
n=39,2.0 \pm 1.3 \\
51 \% \\
\text { Clinician-reported: } \\
n=41,1.9 \pm 0.9 \\
35 \%\end{array}$ & W \\
\hline $\begin{array}{l}\text { Myers and } \\
\text { Myers }^{23}\end{array}$ & $\begin{array}{l}n=31 \text { (18 males, } \\
13 \text { females }) \\
\text { Age } 28.1 \pm 6.7 \mathrm{y}\end{array}$ & ACT (not specified) & $\begin{array}{l}\mathrm{C} / \mathrm{S} \\
\text { General } \\
\text { adherence }\end{array}$ & $\begin{array}{l}\text { Cystic fibrosis adherence } \\
\text { patient questionnaire: } \\
\text { Likert scale, } 1-7 \\
(<4=\text { poor, } 4=\text { average, } \\
>4=\text { high })\end{array}$ & $\begin{array}{l}\text { Mean } \pm \text { SD } \\
3.00 \pm 2.59\end{array}$ & W \\
\hline Ward et al ${ }^{24}$ & $\begin{array}{l}n=117(64 \text { males, } \\
53 \text { females }) \\
\text { Age } 36.3 \pm 19.8 \mathrm{mo}\end{array}$ & CPT (not specified) & $\begin{array}{l}\mathrm{C} / \mathrm{S} \\
\text { General } \\
\text { adherence }\end{array}$ & $\begin{array}{l}\text { Care giver questionnaire: } \\
\text { Likert scale, } 1-7 \\
(\geq 4=\text { adherence is a } \\
\text { problem males, }<4=\text { not a } \\
\text { problem) } \\
\text { (1) } n(\%) \text { with adherence } \\
\text { problems }\end{array}$ & (1) $50.4 \%$ & W \\
\hline Dalcin et $\mathrm{al}^{25}$ & $\begin{array}{l}n=38 \text { (18 males, } \\
20 \text { females }) \\
\text { Age } 23.8 \pm 6.5 \mathrm{y}\end{array}$ & $\begin{array}{l}\text { Respiratory therapy } \\
\text { (not specified) }\end{array}$ & $\begin{array}{l}\mathrm{C} / \mathrm{S} \\
\text { General } \\
\text { adherence }\end{array}$ & $\begin{array}{l}\text { Patient-reported questionnaire: } \\
\text { (1) } n(\%) \text { with } \geq 70 \% \\
\text { overall adherence } \\
\text { (2) Median overall adherence } \\
\text { (3) Each treatment: } n(\%) \\
\text { with high adherence } \\
\text { ( } \geq 5 \mathrm{~d} / \mathrm{wk}=\text { high) } \\
\text { Clinician-reported: } \\
\text { (4) Overall \% median } \\
\text { adherence }\end{array}$ & $\begin{array}{l}\text { Patient-reported } \\
\text { (1) } n=31(81.6 \%) \\
\text { (2) } 79 \% \\
\text { (3) } 84.2 \% \\
\text { (4) Median } 71\end{array}$ & $\mathrm{~W}$ \\
\hline $\begin{array}{l}\text { Myers \& } \\
\text { Horn }^{26}\end{array}$ & $\begin{array}{l}n=563 \text { ( } 253 \text { males, } \\
\quad 310 \text { females) } \\
\text { Mean age } 27.85 \text { y } \\
\text { Range } 18-66 \text { y } \\
\text { Median } 26 \text { y }\end{array}$ & CPT (not specified) & $\begin{array}{l}\mathrm{C} / \mathrm{S} \\
\text { General } \\
\text { adherence }\end{array}$ & $\begin{array}{l}\text { Patient questionnaire: } \\
\text { Likert scale, } 1 \text { (never) to } 5 \\
\text { (always) } \\
\text { (1) } n(\%) \text { who reported being } \\
\text { prescribed daily CPT } \\
\text { (2) } n(\%) \text { with high } \\
\text { adherence ( } \geq 4 \text { on scale) }\end{array}$ & $\begin{array}{l}\text { (1) } n=522 \\
\quad(92.7 \%) \\
\text { (2) } 44 \%\end{array}$ & W \\
\hline $\begin{array}{l}\text { Arias Llorente } \\
\text { et } \mathrm{al}^{27}\end{array}$ & $\begin{array}{l}n=34 \text { ( } 13 \text { males, } \\
\quad 21 \text { females }) \\
\text { Age } 14.5 \mathrm{y} \\
\text { Range } 1.6-40.6 \mathrm{y}\end{array}$ & CPT (not specified) & $\begin{array}{l}\mathrm{C} / \mathrm{S} \\
\text { General } \\
\text { adherence }\end{array}$ & $\begin{array}{l}\text { (1) Self-reported questionnaire } \\
\text { (patient-reported if }>12 \mathrm{y} \text {, } \\
\text { parent-reported if }<12 \mathrm{y} \text { ) } \\
\text { (2) Clinician opinion of } \\
\text { patient's compliance, } \\
\text { scored out of } 8 \text { (compliant } \\
\text { if score was } \geq 4 \text { ) }\end{array}$ & $\begin{array}{l}\text { (1) Questionnaire: } \\
\text { mean adherence } \\
41.2 \% \\
\text { (2) Clinician: mean } \\
\text { adherence } 35.3 \%\end{array}$ & $\mathrm{~W}$ \\
\hline Hobbs et $\mathrm{al}^{28}$ & $\begin{array}{l}n=27 \text { (mothers) } \\
\text { Childs' age range } \\
2-18 \text { y }\end{array}$ & CPT (not specified) & $\begin{array}{l}\mathrm{C} / \mathrm{S} \\
\text { General } \\
\text { adherence }\end{array}$ & $\begin{array}{l}\text { Parent-reported MNCQ: } \\
\text { Treatments completed/ } \\
\text { prescribed) } \times 100\end{array}$ & $\begin{array}{l}\text { Overall adherence: } \\
90.1 \% \\
\text { Individual treatment } \\
\text { CPT: } 91.2 \%\end{array}$ & $\mathrm{~W}$ \\
\hline Abbott et $\mathrm{al}^{29}$ & $\begin{array}{l}n=60 \text { ( } 35 \text { males, } \\
25 \text { females }) \\
\text { Mean age } 20.98 \text { y } \\
\text { Range } 16-44 \text { y }\end{array}$ & CPT (not specified) & $\begin{array}{l}\mathrm{C} / \mathrm{S} \\
\text { General } \\
\text { adherence }\end{array}$ & $\begin{array}{l}\text { Interview, patient-reported } \\
\text { questionnaire: } \\
\text { Degree of adherence on } \\
\text { 6-point scale (adherence } \\
=\geq 4 \text { ) }\end{array}$ & $\begin{array}{l}n(\%) \text { of patients who } \\
\text { were adherent } / n \\
\text { who participated in } \\
\text { treatment: } 32(53 \%)\end{array}$ & M \\
\hline White et al ${ }^{30}$ & $\begin{array}{l}n=57 \text { ( } 34 \text { males, } \\
23 \text { females }) \\
\text { Age } 25.8 \pm 6.8 \mathrm{y}\end{array}$ & $\begin{array}{l}\text { PEP therapy, coughing, } \\
\text { ACBT, huffing, } \\
\text { autogenic drainage, } \\
\text { percussion, PD, flutter } \\
\text { therapy, vibrations }\end{array}$ & $6 \mathrm{mo}$ & $\begin{array}{l}\text { Patient questionnaire (based } \\
\text { on MCFQ) }\end{array}$ & $\begin{array}{l}n(\%) \text { of patients } \\
\quad \text { classed as adherent: } \\
n=40(72 \%)\end{array}$ & $\mathrm{W}$ \\
\hline
\end{tabular}


Table 2. Continued

\begin{tabular}{|c|c|c|c|c|c|c|}
\hline Authors & Participants & Treatment Category & Study Duration & Measurement of Adherence & Results & Q \\
\hline White et $\mathrm{al}^{31}$ & $\begin{array}{l}n=53(22 \text { males, } \\
31 \text { females }) \\
\text { Age } 12.4 \pm 2.57 \mathrm{y}\end{array}$ & $\begin{array}{l}\text { (1) CPT } \\
\text { (2) PEP mask/flutter } \\
\text { coughing, ACBT, } \\
\text { chest wall percussion, } \\
\text { chest wall vibrations }\end{array}$ & $3 d$ & $\begin{array}{l}\text { CCFMP questionnaire: } \\
\text { (1) Patient-reported adherence } \\
\text { to prescription } \\
\text { (2) Parent-reported adherence } \\
\text { to prescription }\end{array}$ & $\begin{array}{l}\text { Reported adherence to } \\
\text { prescriptions: } \\
\text { (1) Patient \%, } \\
66,57 \\
\text { (2) Parent } \%, 55,61\end{array}$ & W \\
\hline $\begin{array}{c}\text { DeLambo } \\
\text { et }^{10}{ }^{10}\end{array}$ & $\begin{array}{l}n=96(50 \text { males } \\
46 \text { females }) \\
\text { Age } 13.12 \pm 1.69 y\end{array}$ & ACT (not specified) & $2 \mathrm{wk}$ & $\begin{array}{l}\text { TARS questionnaire on } \\
\text { following one's treatment } \\
\text { regime: } \\
\text { Likert scale, } 1 \text { (never) to } 5 \\
\text { (always) } \\
\text { (1) Mother-reported } \\
\text { (2) Patient-reported } \\
\text { (3) Father-reported }\end{array}$ & $\begin{array}{l}\text { Mean } \pm \text { SD } \\
\text { (1) } 3.84 \pm 0.98 \\
\text { (2) } 4.14 \pm 0.9 \\
\text { (3) } 4.12 \pm 0.81\end{array}$ & $\mathrm{~W}$ \\
\hline Modi et $\mathrm{al}^{3}$ & $\begin{array}{l}n=37 \text { (19 males, } \\
18 \text { females }) \\
\text { Age } 10.1 \pm 2.5 \mathrm{y}\end{array}$ & ACT (not specified) & $2 \mathrm{wk}$ & $\begin{array}{l}\text { Multimethod assessment: } \\
\text { (1) DMI-CF patient-reported } \\
\text { questionnaire } \\
\text { (2) DMI-CF parent-reported } \\
\text { questionnaire } \\
\text { (3) } 2 \times \text { daily phone diaries by } \\
\text { primary caretaker }\end{array}$ & $\begin{array}{l}\text { Mean } \pm \text { SD } \% \\
\text { adherence } \\
\text { (1) } 66.9 \pm 30.2 \\
\text { (2) } 74.4 \pm 35.3 \\
\text { (3) } 51.1 \pm 40.2\end{array}$ & W \\
\hline $\begin{array}{l}\mathrm{Q}=\text { quality } \\
\mathrm{W}=\text { weak } \\
\mathrm{M}=\text { moderate } \\
\mathrm{CPT}=\text { chest phy } \\
\mathrm{ACT}=\text { airway cl} \\
\mathrm{PEP}=\text { positive e } \\
\mathrm{ACBT}=\text { airway } \\
\mathrm{PD}=\text { postural dr } \\
\mathrm{C} / \mathrm{S}=\text { cross-secti } \\
\text { MNCQ = medica } \\
\text { CCFMP = confid } \\
\text { MCFQ = Manche } \\
\text { TARS = treatmen } \\
\text { DMI-CF = diseas }\end{array}$ & $\begin{array}{l}\text { therapy } \\
\text { ce techniques } \\
\text { tory pressure } \\
\text { of breathing technique } \\
\text { I nonmedical compliance que } \\
1 \text { cystic fibrosis management } \\
\text { cystic fibrosis questionnaire } \\
\text { herence rating scale } \\
\text { inagement interview-cystic fi }\end{array}$ & $\begin{array}{l}\text { onnaire } \\
\text { file } \\
\text { sis }\end{array}$ & & & & \\
\hline
\end{tabular}

measured as $71 \pm 24.5 \%, 78.5 \pm 22.5 \%$, and $78 \pm 20.25 \%$, respectively. ${ }^{10}$

Deoxyribonuclease. Six studies assessed 298 participants (male, $n=113$; female, $n=143$; unspecified, $n=42$ ). All studies were of low quality. A number of strategies were used to determine adherence. Two studies used pharmacy refill data, ${ }^{35,36} 3$ used participant questionnaires, ${ }^{3,25,31}$ 2 used parent questionnaires, 3,31 one used participant interviews, ${ }^{36}$ one used a collection of empty medication vials, ${ }^{11}$ and one used daily telephone diaries. ${ }^{3}$ Mean adherence ranged from $54 \%$ by participant interview ${ }^{36}$ to $77.8 \pm 44.1 \%$ by patient questionnaire. ${ }^{3}$

\section{Pancreatic Enzyme Replacement Therapy}

Nine studies assessed 518 participants (male, $n=262$; female, $n=256$ ). Seven studies were of low quality, and 2 were of moderate quality. Six studies used participant questionnaires $3,25,27,29,31,37$; 3 used parent questionnaires ${ }^{3,22,31}$; one used participant interviews ${ }^{38}$; one used $7-\mathrm{d}$ recordings ${ }^{22}$; and one used pharmacy refill records, daily telephone diaries, and electronic monitoring via the Medication Event Monitoring System (MEMS SmartCap, AARDEX, Richmond, Virginia). ${ }^{3}$ Mean adherence ranged from $27.4 \pm 22.9 \%$ by daily telephone diary ${ }^{3}$ to $96.5 \%$ by patient report. ${ }^{35}$

Two studies reported a combined result for pancreatic enzyme replacement therapy, electrolytes, and supplements in 151 participants (male, $n=77$; female, $n=74$ ). Both studies were of low quality. One study used caregiver questionnaires, ${ }^{24}$ and the other used participant and clinician questionnaires. ${ }^{27}$ Adherence ranged from $70.4 \%$ to $88.2 \% .{ }^{30}$ In the study by Ward et al, ${ }^{24}$ problematic behavior regarding adherence was reported in $6 \%$ of participants.

One low-quality study reported a combined result for antibiotics and pancreatic enzyme replacement therapy in 41 participants (male, $n=20$; female, $n=21$ ) by parent and clinician questionnaires. Mean adherence ranged from $87.5 \pm 17.5 \%$ by clinician report to $95 \pm 15 \%$ by participant report. ${ }^{41}$ 
Adherence to Cystic Fibrosis Programs

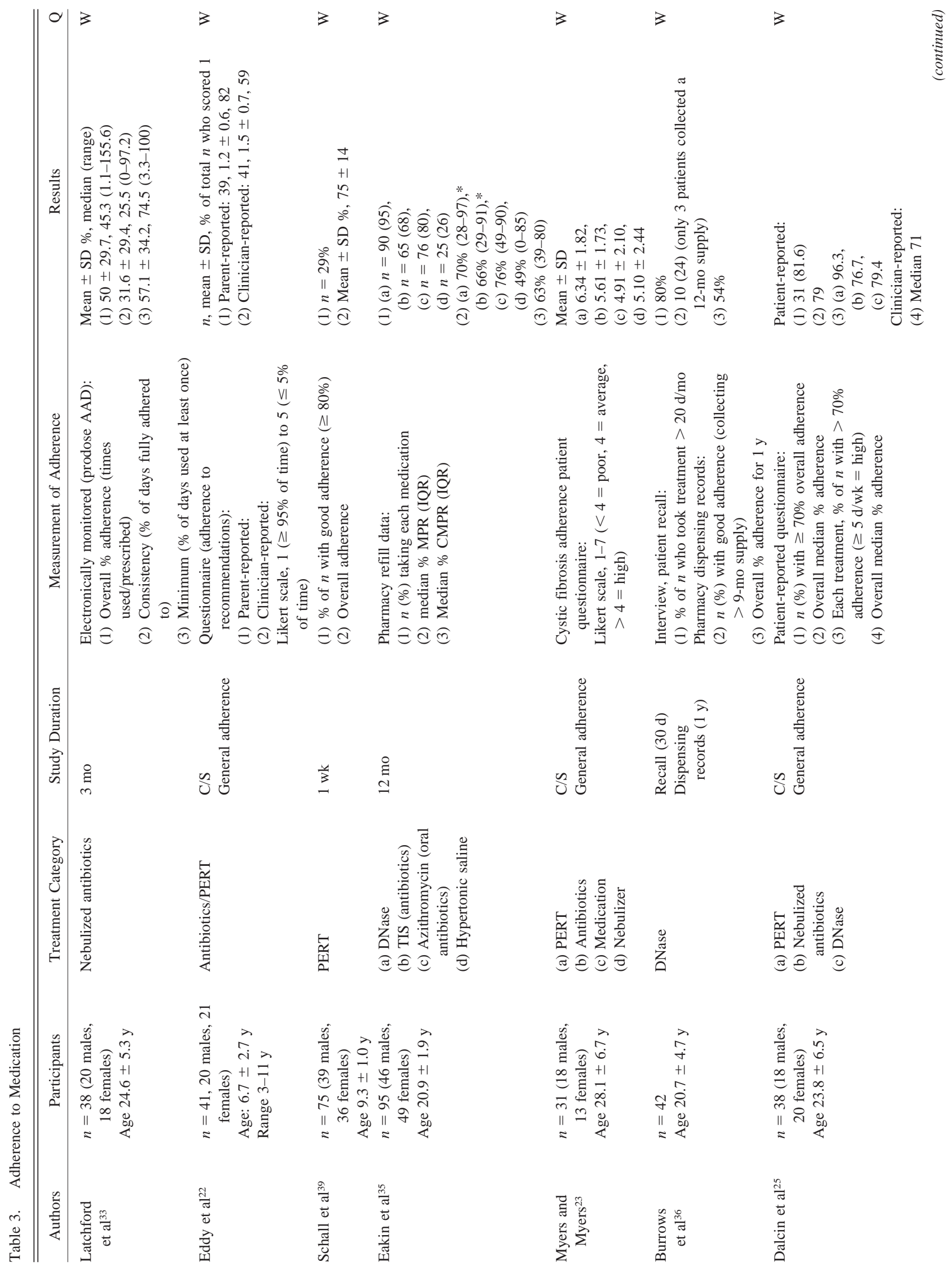




\section{Adherence to Cystic Fibrosis Programs}

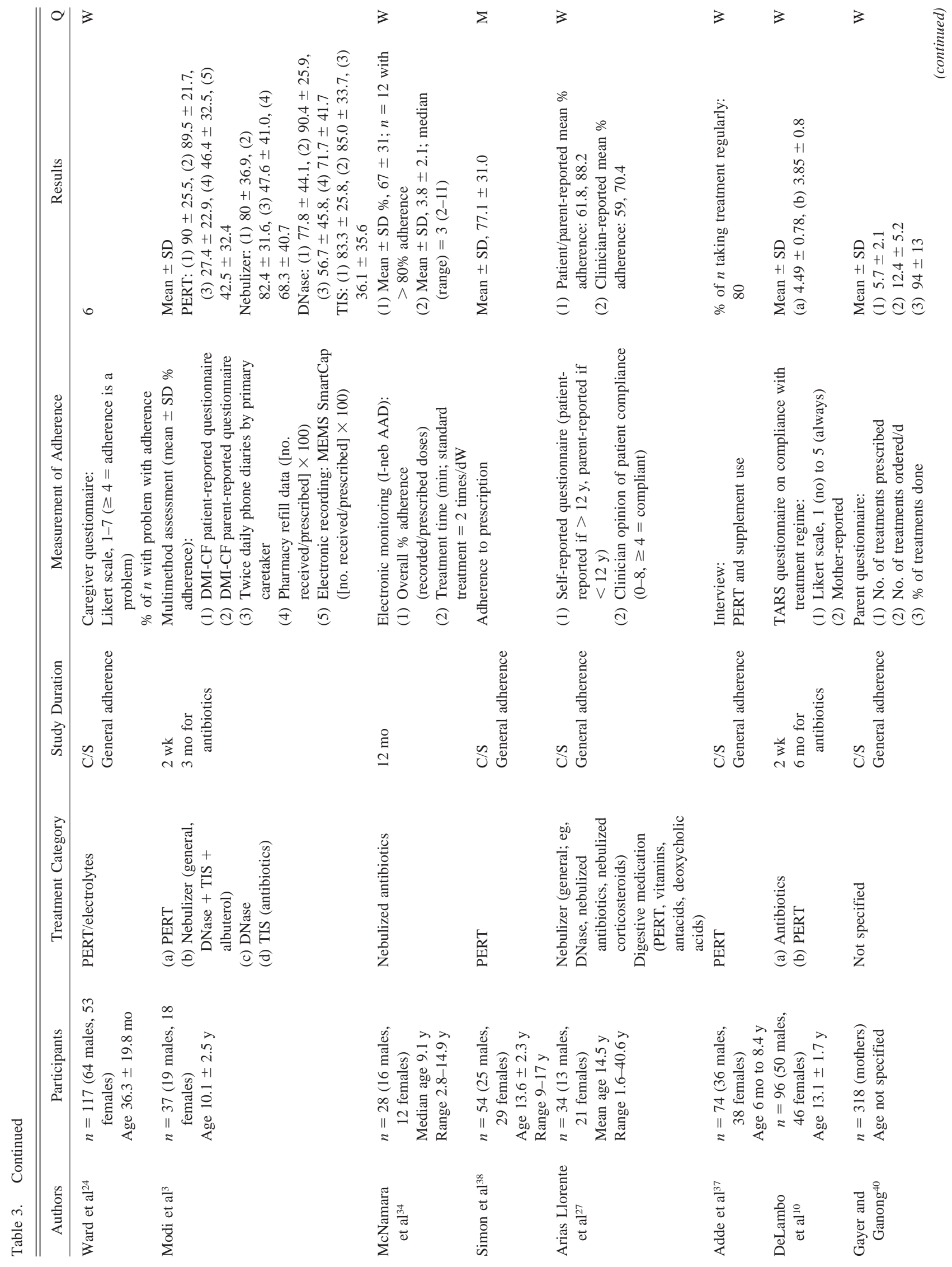


Adherence to Cystic Fibrosis Programs

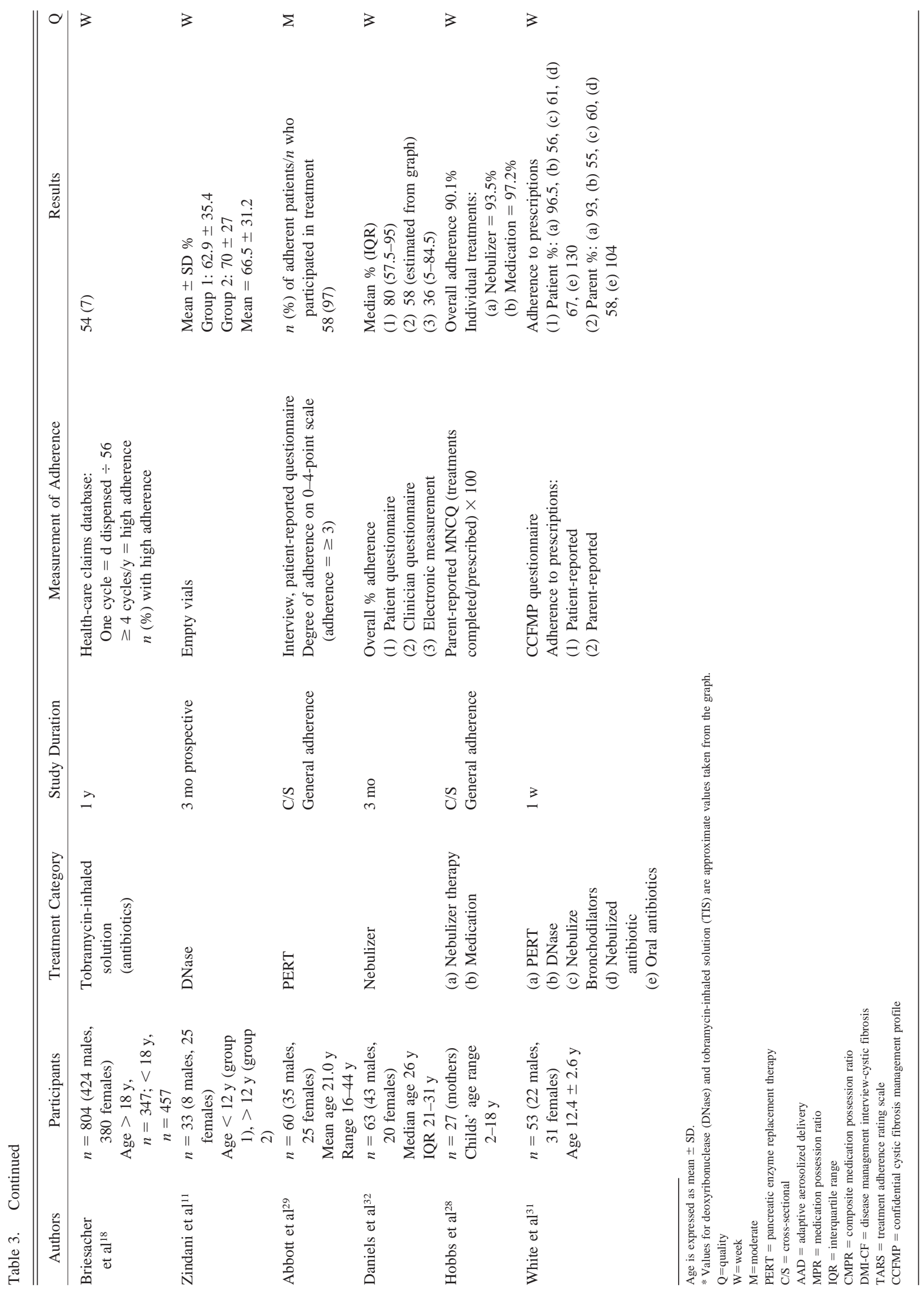


Table 4. Adherence to Nutritional Recommendations

\begin{tabular}{|c|c|c|c|c|c|c|}
\hline Authors & Participants & Treatment Category & $\begin{array}{l}\text { Study } \\
\text { Duration }\end{array}$ & $\begin{array}{l}\text { Measurement of } \\
\text { Adherence }\end{array}$ & Results & Q \\
\hline Schall et al ${ }^{39}$ & $\begin{array}{l}n=75(39 \text { males, } \\
36 \text { females }) \\
\text { Age } 9.3 \pm 1.0 y\end{array}$ & $\begin{array}{l}\text { (a) High-calorie diet } \\
\text { (b) Calories from fat }\end{array}$ & $7 \mathrm{~d}$ & $\begin{array}{l}\text { 7-d food record } \\
\text { (a) (1) \% of } n \text { getting } \\
\geq 120 \% \text { EER } \\
\text { (2) Median \% EER } \\
\text { achieved } \\
\text { (b) (3) } \% \text { of } n \text { getting } \\
\geq 40 \% \text { of total calories } \\
\text { from fat sources } \\
\text { (4) Median \% of EER } \\
\text { from fat }\end{array}$ & $\begin{array}{l}\text { (a) (1) } n=39 \% \\
\text { (2) } 115 \% \text { EER } \\
\text { (b) (3) } 28 \% \\
\text { (4) } 37 \% \text { EER }\end{array}$ & $\mathrm{W}$ \\
\hline Adde et $\mathrm{al}^{37}$ & $\begin{array}{l}n=74 \text { ( } 36 \text { males, } \\
38 \text { females }) \\
\text { Age } 6 \text { mo to } 8.4 \mathrm{y}\end{array}$ & $\begin{array}{l}\text { (a) Use of supplements } \\
\text { (b) High-calorie diet }\end{array}$ & $\begin{array}{l}\mathrm{C} / \mathrm{S} \\
\text { General } \\
\quad \text { adherence }\end{array}$ & $\begin{array}{l}\text { 24-h diet recall } \\
\quad \text { (mean } \pm \text { SD \% RDA } \\
\text { intake) }\end{array}$ & $\begin{array}{l}\text { (a) } 33 \\
\text { (b) } 124(42)\end{array}$ & W \\
\hline Eddy et $\mathrm{al}^{22}$ & $\begin{array}{l}n=41 \text { (20 males, } \\
\quad 21 \text { females) } \\
\text { Age } 6.7 \pm 2.67 \mathrm{y} \\
\text { Range } 3-11 \mathrm{y}\end{array}$ & $\begin{array}{l}\text { (a) Eating/avoiding food } \\
\text { determined by } \\
\text { dietician } \\
\text { (b) Vitamins }\end{array}$ & $\begin{array}{l}\mathrm{C} / \mathrm{S} \\
\text { General } \\
\quad \text { adherence }\end{array}$ & $\begin{array}{l}\text { Questionnaire: } \\
\text { Likert scale, } 1(\geq 95 \% \text { of } \\
\text { time) to } 5 \text { ( } \leq 5 \% \text { of } \\
\text { time) } \\
\text { (1) Parent-reported } \\
\text { adherence: } \\
\text { (2) Clinician-reported: }\end{array}$ & $\begin{array}{l}n \text {, mean } \pm \mathrm{SD}, \% \text { of total } \\
n \text { who scored a } 1 \\
\text { (1) Parent-reported: } \\
\text { (a) } 17,2.2 \pm 1.1,12 \\
\text { (b) } 41,1.1 \pm 0.3,93 \\
\text { (2) Clinician-reported } \\
\text { (a) } 41,1.8 \pm 0.8,41 \\
\text { (b) } 41,1.5 \pm 0.8,66\end{array}$ & W \\
\hline Myers and Myers 23 & $\begin{array}{l}n=31 \text { (18 males, } \\
13 \text { females }) \\
\text { Age } 28.05 \pm 6.7 \mathrm{y}\end{array}$ & $\begin{array}{l}\text { (a) High-calorie diet } \\
\text { (b) Vitamins }\end{array}$ & $\begin{array}{l}\mathrm{C} / \mathrm{S} \\
\text { General } \\
\text { adherence }\end{array}$ & $\begin{array}{l}\text { Cystic fibrosis adherence } \\
\text { patient questionnaire: } \\
\text { Likert scale, } 1-7 \\
\quad(<4=\text { poor } \\
>4=\text { high }\end{array}$ & $\begin{array}{l}\text { Mean } \pm \text { SD } \\
\text { (a) } 4.06 \pm 2.00 \\
\text { (b) } 6.13 \pm 1.74\end{array}$ & W \\
\hline Dalcin et al ${ }^{25}$ & $\begin{array}{l}n=38 \text { (18 males, } \\
20 \text { females }) \\
\text { Age } 23.8 \pm 6.5 \mathrm{y}\end{array}$ & $\begin{array}{l}\text { (a) High-calorie diet } \\
\text { (b) Vitamins A, D, E, K }\end{array}$ & $\begin{array}{l}\mathrm{C} / \mathrm{S} \\
\text { General } \\
\text { adherence }\end{array}$ & $\begin{array}{l}\text { Questionnaire: } \\
\text { Patient-reported: } \\
\text { (1) High }(\geq 70 \%) \text { overall } \\
\text { adherence } \\
\text { (2) Overall \% median } \\
\text { adherence } \\
\text { (3) Each treatment, \% of } \\
n \text { with high adherence } \\
(\geq 5 \mathrm{~d} / \mathrm{wk}=\text { high) } \\
\text { Clinician-reported: } \\
\text { (4) Overall \% median } \\
\text { adherence }\end{array}$ & $\begin{array}{l}\text { Patient-reported: } \\
\text { (1) } n=31(81.6 \%) \\
\text { (2) } 79 \\
\text { (3) (a) 65.8, (b) } 79.4 \\
\text { Clinician-reported: } \\
\text { (4) Median } 71\end{array}$ & W \\
\hline Hollander et $\mathrm{al}^{42}$ & $\begin{array}{l}n=79 \text { (44 males, } \\
\quad 35 \text { females; } \\
\text { with PI) } \\
\text { Median age } 25 \mathrm{y} \\
\text { Range } 18-50 \mathrm{y}\end{array}$ & Vitamins A, D, E, K & $\begin{array}{l}\mathrm{C} / \mathrm{S} \\
\text { General } \\
\text { adherence }\end{array}$ & $\begin{array}{l}\text { Telephone survey of } \\
\text { patients Intake vs } \\
\text { recommendations } \\
\text { (1) } \% \text { of } n \text { taking } \\
\text { vitamins } \\
\text { (2) } \% \text { of } n \text { using specific } \\
\text { vitamins } \\
\text { (3) } \% \text { of } n \text { reaching } \\
\text { recommended amounts }\end{array}$ & $\begin{array}{l}\text { (1) } 84 \% \\
\text { Vitamins A, D, E, K (\%) } \\
\text { (2) } 71,72,77,33 \\
\text { (3) } 9,32,59,81\end{array}$ & W \\
\hline Hobbs et $\mathrm{al}^{28}$ & $\begin{array}{l}n=27 \text { (mothers) } \\
\text { Childs' age range } \\
2-18 \text { y }\end{array}$ & High-calorie diet & $\begin{array}{l}\mathrm{C} / \mathrm{S} \\
\text { General } \\
\text { adherence }\end{array}$ & $\begin{array}{l}\text { Parent-reported MNCQ: } \\
\text { Adherence ([treatments } \\
\text { completed/prescribed] } \\
\quad \times 100)\end{array}$ & Nutrition $81 \%$ & W \\
\hline Anthony et $\mathrm{al}^{43}$ & $\begin{array}{l}n=25 \text { ( } 14 \text { males, } \\
\quad 11 \text { females) } \\
\text { Mean age } 9.1 \mathrm{y} \\
\text { Range } 7-12 \mathrm{y}\end{array}$ & High-calorie diet & $7 d$ & $\begin{array}{l}\text { 7-d food weighed records } \\
\% \text { of } n \text { achieving } \\
>120 \% \text { DRI }\end{array}$ & $16 \%$ & W \\
\hline Abbott et al ${ }^{29}$ & $\begin{array}{l}n=60 \text { ( } 35 \text { males, } \\
\quad 25 \text { females) } \\
\text { Mean age } 20.98 \text { y } \\
\text { Range } 16-44 \text { y }\end{array}$ & Vitamins & $\begin{array}{l}\mathrm{C} / \mathrm{S} \\
\text { General } \\
\text { adherence }\end{array}$ & $\begin{array}{l}\text { Interview, patient-reported } \\
\text { questionnaire: } \\
\text { Degree of adherence on } \\
\text { 0-4-point scale } \\
\text { (adherence }=\geq 3 \text { ) }\end{array}$ & $\begin{array}{l}n(\%) \text { patients adherent } / n \\
\text { who participated in } \\
\text { treatment } \\
37(83)\end{array}$ & M \\
\hline
\end{tabular}


Table 4. Continued

\begin{tabular}{|c|c|c|c|c|c|c|}
\hline Authors & Participants & Treatment Category & $\begin{array}{l}\text { Study } \\
\text { Duration }\end{array}$ & Measurement of Adherence & Results & Q \\
\hline Modi et $\mathrm{al}^{3}$ & $\begin{array}{l}n=37 \text { ( } 19 \text { males, } \\
18 \text { females) } \\
\text { Age } 10.1 \pm 2.5 \mathrm{y} \\
\text { Age } 3 \mathrm{~m} \text { to } 56 \mathrm{y}\end{array}$ & Vitamins & $2 \mathrm{wk}$ & $\begin{array}{l}\text { Multimethod assessment: } \\
\text { Mean } \pm \text { SD \% adherence } \\
\text { (1) DMI-CF patient- } \\
\text { reported questionnaire } \\
\text { (2) DMI-CF parent- } \\
\text { reported questionnaire } \\
\text { (3) Twice daily phone } \\
\text { diaries by primary care } \\
\text { taker } \\
\text { (4) Pharmacy refill data } \\
\text { ([no. received/ } \\
\text { prescribed] } \times 100)\end{array}$ & $\begin{array}{l}\text { Mean } \pm \text { SD \% } \\
\text { Vitamin } \\
\text { (1) } 93.8 \pm 17.1 \\
\text { (2) } 88.4 \pm 27.6 \\
\text { (3) } 22.2 \pm 34.2 \\
\text { (4) } 33.7 \pm 45.6\end{array}$ & $\mathrm{~W}$ \\
\hline Borowitz et al ${ }^{41}$ & $\begin{array}{l}n=80 \text { ( } 38 \text { males, } \\
42 \text { females; } \\
\text { with PI })\end{array}$ & Multivitamins & $\begin{array}{l}\mathrm{C} / \mathrm{S} \\
\text { General } \\
\text { adherence }\end{array}$ & $\begin{array}{l}\text { Questionnaire, phone } \\
\text { interview } \\
\% \text { Adherence to prescription }\end{array}$ & $n=47 \%$ & $\mathrm{~W}$ \\
\hline Simon et al ${ }^{38}$ & $\begin{array}{l}n=54(25 \text { males, } \\
29 \text { females }) \\
\text { Age } 13.61 \pm 2.32 \mathrm{y} \\
\text { Range }=9-17 \mathrm{y}\end{array}$ & $\begin{array}{l}\text { (a) High-calorie diet } \\
\text { (b) Calories from fat }\end{array}$ & $\begin{array}{l}\mathrm{C} / \mathrm{S} \\
\text { General } \\
\text { adherence }\end{array}$ & $\begin{array}{l}\text { Interview (24-h diet recall) } \\
\text { (1) (a) Reported caloric } \\
\text { intake } \\
\text { Adherence }=\geq 120 \% \text { DRI } \\
\text { (2) (b) } \% \text { of } n \text { adherent } \\
\text { ( } \geq 35 \% \text { of total calories } \\
\text { from fat) }\end{array}$ & $\begin{array}{l}\text { (mean } \pm \text { SD) } \\
\text { (1) (a) } 3,951 \pm 1,747 \\
\text { Range } 1,172-9,812 ; \% \text { of } \\
n \text { adherent }=76 \\
\text { (2) (b) } 59.3\end{array}$ & M \\
\hline Arias Llorente et al ${ }^{27}$ & $\begin{array}{l}n=34 \text { ( } 13 \text { males, } \\
21 \text { females }) \\
\text { Mean age } 14.5 \\
\text { Range } 1.6-40.6 \mathrm{y}\end{array}$ & High-calorie supplements & $\begin{array}{l}\text { C/S } \\
\text { General } \\
\text { adherence }\end{array}$ & $\begin{array}{l}\text { (1) Self-reported } \\
\text { questionnaire (patient- } \\
\text { reported if }>12 \mathrm{y}, \\
\text { parent-reported if }<12 \mathrm{y} \text { ) } \\
\text { (2) Clinician opinion of } \\
\text { patient compliance (score } \\
0-8 \text {, compliant if } \geq 4 \text { ) }\end{array}$ & $\begin{array}{l}\text { (1) Parent/patient-reported } \\
\text { mean adherence of } 59 \% \\
\text { (2) Clinician-reported } \\
\text { mean adherence of } 56 \%\end{array}$ & W \\
\hline DeLambo et al ${ }^{10}$ & $\begin{array}{l}n=96(50 \text { males, } \\
\quad 46 \text { females }) \\
\text { Age } 13.12 \pm 1.69 \mathrm{y}\end{array}$ & Nutrition & $2 \mathrm{wk}$ & $\begin{array}{l}\text { TARS questionnaire on } \\
\text { following treatment regime: } \\
\text { Likert scale, } 1 \text { (never) to } 5 \\
\text { (always) } \\
\text { (1) Mother-reported } \\
\text { (2) Patient-reported } \\
\text { (3) Father-reported }\end{array}$ & $\begin{array}{l}\text { Mean } \pm \text { SD } \\
\text { (1) } 2.63 \pm 1.27 \\
\text { (2) } 2.82 \pm 1.41 \\
\text { (3) } 2.59 \pm 1.27\end{array}$ & $\mathrm{~W}$ \\
\hline White et al ${ }^{31}$ & $\begin{array}{l}n=53(22 \text { males, } \\
31 \text { females }) \\
\text { Age } 12.4 \pm 2.57 \mathrm{y}\end{array}$ & Tube feeding & $1 \mathrm{wk}$ & $\begin{array}{l}\text { CCFMP questionnaire: } \\
\text { (1) Patient-reported } \\
\text { adherence } \\
\text { (2) Parent-reported } \\
\text { adherence }\end{array}$ & $\begin{array}{l}\text { Adherence to prescriptions. } \\
\text { (1) Patient } 95 \% \\
\text { (2) Parent } 53 \%\end{array}$ & W \\
\hline Zindani et al ${ }^{11}$ & $\begin{array}{l}n=33 \text { (8 males, } 25 \\
\text { females) } \\
\text { Age }<12 \text { y (group } \\
\text { 1) }>12 \text { y (group } \\
\text { 2) }\end{array}$ & Vitamins A, D, E, K & $\begin{array}{l}3 \text { mo } \\
\text { prospective }\end{array}$ & $\begin{array}{l}\text { Electronic recording, MEMS } \\
\text { SmartCap }\end{array}$ & $\begin{array}{l}\text { Group 1: } 70.5 \pm 27.3 \% \\
\text { Group 2: } 56.7 \pm 20.6 \% \\
\text { Mean } 63.6 \pm 24\end{array}$ & $\mathrm{~W}$ \\
\hline $\begin{array}{l}\text { Age is expressed as mean } \pm \\
Q=\text { quality } \\
\mathrm{W}=\text { weak } \\
\mathrm{M}=\text { moderate } \\
\mathrm{C} / \mathrm{S}=\text { cross-sectional } \\
\mathrm{EER}=\text { estimated energy requ } \\
\mathrm{RDA}=\text { recommended daily } \\
\mathrm{MNCQ}=\text { Medical and Nonn } \\
\mathrm{DRI}=\text { daily recommended in } \\
\text { DMI-CF = disease managem } \\
\text { TARS = treatment adherence } \\
\text { CCFMP = confidential cystic } \\
\text { MEMS = medication event-n }\end{array}$ & $\begin{array}{l}\text { SD. } \\
\text { uirement } \\
\text { allowance } \\
\text { nedical Compliance Questionn } \\
\text { take } \\
\text { ent interview-cystic fibrosis } \\
\text { rating scale } \\
\text { c fibrosis management profile } \\
\text { nonitoring system }\end{array}$ & & & & & \\
\hline
\end{tabular}


Table 5. Adherence to Exercise Recommendations

\begin{tabular}{|c|c|c|c|c|c|c|}
\hline Authors & Participants & Treatment Category & Study Duration & Measurement of Adherence & Results & Q \\
\hline $\begin{array}{l}\text { Myers and } \\
\text { Myers }^{23}\end{array}$ & $\begin{array}{l}n=31(18 \text { males, } \\
13 \text { females }) \\
\text { Age } 28.1 \pm 6.7 \mathrm{y}^{*}\end{array}$ & Regular exercise & $\begin{array}{l}\mathrm{C} / \mathrm{S} \\
\text { General adherence }\end{array}$ & $\begin{array}{l}\text { Cystic fibrosis adherence patient } \\
\text { questionnaire: } \\
\text { Likert scale, } 1-7(<4=\text { poor, } \\
4=\text { average, }>4=\text { high })\end{array}$ & $4.41 \pm 1.87^{*}$ & $\mathrm{~W}$ \\
\hline White et $\mathrm{a}^{30}$ & $\begin{array}{l}n=57 \text { ( } 34 \text { males, } \\
23 \text { females }) \\
\text { Age } 25.8 \pm 6.8 y^{*}\end{array}$ & $\begin{array}{l}\text { Exercise (walking, } \\
\text { gym) }\end{array}$ & $6 \mathrm{mo}$ & $\begin{array}{l}\text { Patient questionnaire (based on } \\
\text { MCFQ) }\end{array}$ & $\begin{array}{l}n(\%) \text { of subjects } \\
\quad \text { classed as adherent } \\
n=24(42.1 \%)\end{array}$ & $\mathrm{W}$ \\
\hline Hobbs et $\mathrm{al}^{28}$ & $\begin{array}{l}n=27 \text { (mothers) } \\
\text { Childs' age range } \\
2-18 \text { y }\end{array}$ & Exercise (not specified) & $\begin{array}{l}\mathrm{C} / \mathrm{S} \\
\text { General adherence }\end{array}$ & $\begin{array}{l}\text { Parent-reported MNCQ: } \\
\text { Adherence }(\text { treatments } \\
\quad \text { completed/prescribed] } \times 100 \text { ) }\end{array}$ & Exercise $=88 \%$ & $\mathrm{~W}$ \\
\hline Abbott et al ${ }^{29}$ & $\begin{array}{l}n=60 \text { ( } 35 \text { males, } \\
25 \text { females }) \\
\text { Median age } 20.98 \text { y } \\
\quad(\text { range } 16-44 \text { y) }\end{array}$ & Exercise (not specified) & $\begin{array}{l}\mathrm{C} / \mathrm{S} \\
\text { General adherence }\end{array}$ & $\begin{array}{l}\text { Interview, patient-reported } \\
\text { questionnaire: } \\
\text { Degree of adherence ( } 3 \\
\text { questions assessed by } \\
\text { physiotherapist) }\end{array}$ & $\begin{array}{l}n(\%) \text { of subjects } \\
\text { who were adherent } / n \\
\text { who participated in } \\
\text { the treatment } \\
\quad 41(75 \%)\end{array}$ & M \\
\hline Dalcin et $\mathrm{a}^{25}$ & $\begin{array}{l}n=38(18 \text { males } \\
20 \text { females }) \\
\text { Age } 23.8 \pm 6.5 \mathrm{y}^{*}\end{array}$ & $\begin{array}{l}\text { Exercise (physical } \\
\text { activity) }\end{array}$ & $\begin{array}{l}\mathrm{C} / \mathrm{S} \\
\text { General adherence }\end{array}$ & $\begin{array}{l}\text { Patient-reported questionnaire: } \\
\text { (1) } \mathrm{N}(\%) \text { with high }(\geq 70 \%) \\
\text { overall adherence } \\
\text { (2) Median overall adherence } \\
\text { (3) Each treatment ( } \% \text { of } n \text { with } \\
\text { high adherence, } \geq 5 \mathrm{~d} / \mathrm{wk}) \\
\text { Clinician-reported: } \\
\text { (4) Overall median adherence }\end{array}$ & $\begin{array}{l}\text { Patient-reported: } \\
\text { (1) } n=31(81.6 \%) \\
\text { (2) } 79 \% \\
\text { (3) } 21.1 \% \\
\text { Clinician-reported: } \\
\text { (4) Median }=71 \%\end{array}$ & $\mathrm{~W}$ \\
\hline \multicolumn{7}{|c|}{$\begin{array}{l}\text { *Values are expressed as mean } \pm S D \\
Q=\text { quality } \\
W=\text { weak } \\
M=\text { moderate } \\
C / S=\text { cross-sectional } \\
M C F Q=\text { Manchester cystic fibrosis questionnaire } \\
\text { MNCQ = medical and nonmedical compliance ques }\end{array}$} \\
\hline
\end{tabular}

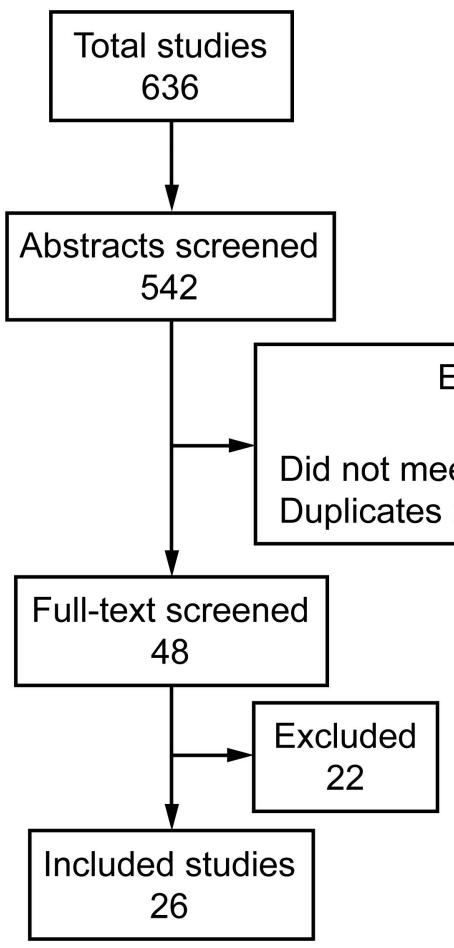

Fig. 1. Flow chart of study selection process.
Two studies assessed adherence rates for medications in general in 58 participants (male, $n=18$; female, $n=13$; unspecified, $n=27$ ). One used participant questionnaires, ${ }^{26}$ and the other used parent questionnaires. ${ }^{28}$ Mean adherence ranged from $70.1 \pm 30 \%{ }^{26}$ to $97.2 \% .{ }^{28}$

\section{Nutrition}

Sixteen papers assessed 837 participants' adherence to various nutritional recommendations (male, $n=399$; female, $n=411$; unspecified, $n=27$ ). Fourteen of the studies were of low quality, and 2 were of high quality (Table 4).

Adherence to calorie intake was assessed in 10 studies with 507 participants (male, $n=235$; female, $n=245$; unspecified, $n=27$ ). All but one study was of low quality. Five studies used participant questionnaires, ,3,25,27,31 3 used parent questionnaires, ${ }^{10,28,31}$ one used clinician questionnaires, ${ }^{28} 2$ used 24-h recall, ${ }^{37,38}$ and 2 used 7-d food records. ${ }^{39,43}$ Reported adherence to guidelines ranged from $40.8 \pm 31.75 \% \%^{10}$ to $81 \% .{ }^{28}$

Adherence to vitamin intake was assessed in 8 studies with 399 participants (male, $n=200$; female, $n=199$ ). 
All but one study was of low quality. Four studies used participant questionnaires, $, 3,23,25,292$ used parent questionnaires, 3,22 one used clinician questionnaires, 223 used interviews via telephone, ${ }^{3,41,42}$ and one used pharmacy refill data and electronic recordings via MEMS SmartCap. ${ }^{3}$ Mean adherence ranged from $22.2 \pm 34.2 \%$ by daily telephone diary $^{3}$ to $97.5 \pm 7.5 \%$ by parent questionnaire. ${ }^{22}$ In another study, the percentages of the sample group who reached the recommended doses for vitamins A, D, E, and K were $9,32,59$, and $81 \%$, respectively. ${ }^{42}$

One low-quality study assessed compliance with a dietitian's food guidelines. Forty-one participants (male, $n=20$; female, $n=21$ ) were included. Mean adherence ranged from $70 \pm 27.5 \%$ by clinician questionnaire to $80 \pm 20 \%$ by parent questionnaire..$^{22}$

\section{Airway Clearance Techniques}

Eleven papers assessed 1058 participants (male, $n=$ 496; female, $n=535$; unspecified, $n=27$ ). All but one study was of low quality. Eight studies used participant questionnaires, 3,4,23,25-27,30,31 4 used parent questionnaires, ${ }^{3,22,28,30}, 2$ used clinician questionnaires, ${ }^{22,27}$ one used caregiver questionnaires, ${ }^{24}$ and one used daily telephone diaries. ${ }^{3}$ Only 2 papers of low quality ${ }^{30,31}$ specified the individual aspects of chest physical therapy involved (positive expiratory pressure, coughing, active cycle of breathing technique, chest wall percussion, chest wall vibrations). All other papers $(n=10)$ simply summarized treatment as airway clearance techniques or chest physical therapy. Adherence rates ranged from $33.3 \pm 43.15 \%^{23}$ to $91.2 \%^{28}$ by patient questionnaire.

\section{Exercise}

Five papers assessed 321 participants (male, $n=213$; female, $n=81$; unspecified, $n=27$ ). All papers were of low quality. Four studies used participant questionnaires, ${ }^{23,25,29,30}$ and one study used parent questionnaires. ${ }^{28}$ Only 2 papers described the exercise undertaken: one paper described exercise as walking or attending a gym, ${ }^{28}$ whereas the second reported exercise as physical activity. ${ }^{25}$ Details of exercise prescriptions were not included. All other papers simply reported the category as exercise, and limited details to reporting compliance rates to regular exercise, number of times prescribed exercises were undertaken, or whether exercises were undertaken every day or almost every day (Table 5).

\section{Discussion}

This systematic review examined levels of adherence to home treatment programs for people with CF. Twenty-six studies were included and categorized into one of four main themes: exercise, medication, airway clearance, and nutrition. Results showed that adherence levels were generally low and wide ranging (nutrition, 22.2-97.5\%; airway clearance techniques, 33.3-91.2\%; exercise, 56.888\%; pancreatic enzyme replacement therapy, 27.4-96.5\%; medication [inhaled therapy], 31.6-85\%), varying greatly depending on the type of treatment being assessed and the mode of assessment being employed.

The present study included home-based observational studies only to establish subjects' own level of adherence, which is an integral part of the long-term management of $\mathrm{CF}$. Intervention studies were excluded due to the effect of participating in a formal research trial, in which greater monitoring/measurement of patients would take place. Indeed, compared with a previous review of randomized controlled trials examining adherence rates for airway clearance techniques (88-96\%), ${ }^{14}$ rates for compliance in observational studies reported in the present study are lower (33.3-91.2\%). The previous review of adherence to antibiotics (mixed methodologies) reported similar adherence rates $(35-87 \%)^{13}$ compared with the present review (36$85 \%)$.

Multiple issues concerning the design of studies investigating adherence were found: study duration, participant recall, and use of multiple measures and questionnaires.

The time frame over which the studies were conducted varied greatly from 2 weeks ${ }^{37}$ to 6 months, ${ }^{31}$ which could influence recall bias. Given that $\mathrm{CF}$ is a chronic disease, one would argue that studies of longer duration are more clinically relevant. Shorter intervals have been used previously because of the assumption that recall would be better for shorter periods ${ }^{44}$; however, it has been reported that patients do not recall more recent pill-taking events any more accurately. ${ }^{44,45}$

Some studies utilized multiple modes of assessment, ${ }^{3,30,37}$ which may, in some cases, prove challenging for participants when multiple outcome scales are used (eg, Likert scale, percentage rates, etc). Issues with health literacy and numeracy may impact on patients' ability to complete adherence forms when different scoring scales are utilized (eg, percentages) and need to be considered. ${ }^{46}$

A large range of tools (electronic technologies, selfreported questionnaires) were also utilized to determine levels of adherence. Daily telephone diaries have been thought to allow for a greater precision of recalled information, ${ }^{47}$ and electronic recording via MEMS SmartCap allows for precise recording of usage. ${ }^{48}$

Disparities between self-reported and objective measures of medication adherence were found; both adherence to nebulized antibiotics by parent and self-reported adherence were higher compared with electronic monitoring, consistent with previous research. ${ }^{3,10}$ However, one must be cautious when interpreting results given the low-quality rating of the majority of studies. The improvement in pa- 
tient-reported adherence to treatments and the availability of objective recordings in clinical practice (eg, measuring changes in sweat chloride in those taking ivacaftor ${ }^{49}$ ) should allow for open and honest discussion between patient and clinician. ${ }^{32}$ Similar disparities have been found in other chronic conditions in which self-reported measures are higher than objective measures (eg, functional ability of patients with low back pain ${ }^{50}$ and adherence to medication for patients with HIV ${ }^{51-53}$ ).

Mismatches were also found regarding adherence rates depending on who completed the questionnaire. When comparing people with $\mathrm{CF}$ and their health-care professionals (physiotherapist or doctor), health-care professionals' opinion/impression of adherence was lower than that of the people with CF, similar to levels reported for adults with hypertension. ${ }^{54}$ Multiple factors have been reported as reasons for this (eg, impact of a patient's previous medical history ${ }^{55}$ or a patient's expectations of the consultation ${ }^{56}$ ). Self-reported adherence levels were also found to be generally greater than those reported by parents. ${ }^{3,22,25,32}$ White et $\mathrm{al}^{31}$ found a $30 \%$ disparity between $\mathrm{CF}$ teams' recommendations for oral antibiotics and CF patients' reports, compared with a $4 \%$ disparity for parents. Similarly with regard to adherence with tube feeding, patients with $\mathrm{CF}$ reported 95\% adherence compared with the parents' report of 53\% despite both groups being aware of the CF teams' recommendations. ${ }^{31}$ Along with issues regarding recall, patients with $\mathrm{CF}$ and respondents may provide answers that conform to their perceived expectations of their interviewer, as seen in patients with HIV. 57,58

The challenge for researchers in measuring adherence rates is first to define adherence. The WHO defines adherence as the extent to which a person's behavior (in terms of taking medication, following diets, or executing lifestyle changes) coincides with medical or health advice. ${ }^{59}$ However, adherence to a treatment plan has many levels, such as overall adherence (defined as times treatment is used compared with times prescribed), adherence to the full-length treatment, and the number of days the treatment is used..$^{33}$ These multiple factors relating to adherence again make it difficult to establish, and measuring it is an inexact science. ${ }^{6}$

Reasons for non-adherence to CF have also been explored. As survival rates improve, people with $\mathrm{CF}$ are faced with new challenges relating to age, such as the transition between adolescence to adulthood and coming to terms with their independence with regard to adherence. ${ }^{28}$ It is therefore not surprising that they are nonadherent to aspects of their treatment regimen at different times throughout their lives. This behavior can be acknowledged as a normal adaptation to the physical and emotional intrusion of illness. ${ }^{6}$ For a person growing up with $\mathrm{CF}$, treatment demands become repetitive, often with no immediate perceived benefit. ${ }^{30}$ These young adults are de- termined to live as normal a life as possible and acknowledge that sometimes they are making an informed decision not to carry out their treatment. Older children and adults report poor adherence to treatment regimens, describing them as boring, different from the normal lives of their peers, and a waste of time. ${ }^{27}$

However, it cannot be assumed that reduction in administration time alone will solve the adherence dilemma. For example, asthma medications take only a few seconds to administer via a metered-dose inhaler or powder inhaler, yet adherence rates are low. Treatment programs should perhaps be planned around individual patient's requirements, modifying treatment ideals when necessary according to the exigency and pattern of that patient's lifestyle. ${ }^{60}$

Innovative approaches to increase patients' adherence to recommendations are required. The use of mobile health technologies and telecommunication has rapidly been integrated into the health-care delivery system for the management of chronic illnesses (eg, hypertension, ${ }^{61}$ weight loss, ${ }^{62}$ and diabetes ${ }^{63}$ ) and may prove useful for improving adherence in patients with $\mathrm{CF}$ by allowing adolescents to directly call their CF health-care team and by promoting safe social interactions and peer support. ${ }^{4}$

The results of this systematic review should be considered in light of the following limitations. Only papers written in English and published in the last $20 \mathrm{y}$ were included to reflect the development of guidelines and best practice for the management of patients with $\mathrm{CF}$.

\section{Conclusion}

Overall adherence to home treatments was assessed with a variety of adherence tools in low-quality studies. There were wide disparities in adherence rates, with studies utilizing self-reported tools consistently higher than those utilizing objective measures. The lower objective measures of adherence suggest current efforts to improve adherence are appropriate.

\section{ACKNOWLEDGMENTS}

We thank Ms Anastasia Kitova, who was involved in the search strategy of the review.

\section{REFERENCES}

1. Baum A, Newman S, Weinman J, West R, McManus C. The Cambridge handbook of psychology, health and medicine. Cambridge: Cambridge University Press; 1997.

2. Cystic Fibrosis Foundation Patient Registry 2011 Annual Data Report. Cystic Fibrosis Foundation; 2012. http://www.cff.org/uploaded files/research/clinicalresearch/2011-patient-registry.pdf. Accessed October 30, 2011.

3. Modi AC, Lim CS, Yu N, Geller D, Wagner MH, Quittner AL. A multi-method assessment of treatment adherence for children with cystic fibrosis. J Cyst Fibros 2006;5(3):177-185. 


\section{Adherence to Cystic Fibrosis Programs}

4. Marciel KK, Saiman L, Quittell LM, Dawkins K, Quittner AL. Cell phone intervention to improve adherence: cystic fibrosis care team, patient, and parent perspectives. Pediatr Pulmonol 2010;45(2):157-164.

5. Sawicki GS, Sellers DE, Robinson WM. High treatment burden in adults with cystic fibrosis: challenges to disease self-management. J Cyst Fibros 2009;8(2):91-96.

6. Geller DE, Madge S. Technological and behavioral strategies to reduce treatment burden and improve adherence to inhaled antibiotics in cystic fibrosis. Respir Med 2011;105(Suppl 2):S24-S31.

7. Cockburn J, Gibberd RW, Reid AL, Sanson-Fisher R. Determinants of noncompliance with short-term antibiotic regimens. BMJ 1987; 295(6602):814-818.

8. Elkins MR, Robinson M, Rose BR, Harbour C, Moriarty CP, Marks $\mathrm{GB}$, et al. A controlled trial of long-term inhaled hypertonic saline in patients with cystic fibrosis. N Engl J Med 2006;354(3):229-240.

9. Quinn J, Latchford G, Duff A, Conner M, Pollard K, Morrison L, et al. Measuring, predicting and improving adherence to inhalation in patients with CF: a randomised controlled study of motivational interviewing. Pediatr Pulmonol 2004;38(S27):360.

10. DeLambo KE, Ievers-Landis CE, Drotar D, Quittner AL. Association of observed family relationship quality and problem-solving skills with treatment adherence in older children and adolescents with cystic fibrosis. J Pediatr Psychol 2004 29(5):343-353.

11. Zindani GN, Streetman DD, Streetman DS, Nasr SZ. Adherence to treatment in children and adolescent patients with cystic fibrosis. J Adolesc Health 2006;38(1):13-17.

12. Passero MA, Remor B, Salomon J. Patient-reported compliance with cystic fibrosis therapy. Clin Pediatr 1981;20(4):264-268.

13. Weiner JR, Toy EL, Sacco P, Duh MS. Costs, quality of life and treatment compliance associated with antibiotic therapies in patients with cystic fibrosis: a review of the literature. Expert Opin Pharmacother 2008;9(5):751-766.

14. Main E, Prasad A, van der Schans CP. Conventional chest physiotherapy compared to other airway clearance techniques for cystic fibrosis. Cochrane Database Syst Rev 2005:(1):CD002011.

15. Berg JS, Dischler J, Wagner DJ, Raia JJ, Palmer-Shevlin N. Medication compliance: a healthcare problem. Ann Pharmacother 1993; 27(9 Suppl):S1-S24.

16. Bauman LJ, Wright E, Leickly FE, Crain E, Kruszon-Moran D, Wade SL, Visness CM. Relationship of adherence to pediatric asthma morbidity among inner-city children. Pediatrics 2002;110(1 Pt 1):e6.

17. Weiss KB, Gergen PJ, Hodgson TA. An economic evaluation of asthma in the United States. N Engl J Med 1992;326(13):862-866.

18. Briesacher BA, Quittner AL, Saiman L, Sacco P, Fouayzi H, Quittell LM. Adherence with tobramycin inhaled solution and health care utilization. BMC Pulm Med 2011;11:5.

19. de Abreu Silva FA, Dodge JA. Guidelines for the diagnosis and management of cystic fibrosis. World Health Organization, 1996. http://www.cfww.org/who/article/198/guidelines_for_the_diagnosis_ and_management_of\%20cystic_fibrosis. Accessed June 6, 2014.

20. Thomas BH, Ciliska D, Dobbins M, Micucci S. A process for systematically reviewing the literature: providing the research evidence for public health nursing interventions. Worldviews Evid Based Nurs 2004;1(3):176-184.

21. Deeks JJ, Dinnes J, D’Amico R, Sowden AJ, Sakarovitch C, Song F, et al. Evaluating non-randomised intervention studies. Health Technol Assess 2003;7(27)::iii-x,1-173.

22. Eddy ME, Carter BD, Kronenberger WG, Conradsen S, Eid NS, Bourland SL, Adams G. Parent relationships and compliance in cystic fibrosis. J Pediatr Health Care 1998;12(4):196-202.

23. Myers LB, Myers F. The relationship between control beliefs and self-reported adherence in adults with cystic fibrosis. Psychol Health Med 1999;4(4):387-391.
24. Ward C, Massie J, Glazner J, Sheehan J, Canterford L, Armstrong D, et al. Problem behaviours and parenting in preschool children with cystic fibrosis. Arch Dis Child 2009;94(5):341-347.

25. Dalcin Pde T, Rampon G, Pasin LR, Ramon GM, Abrahão CL, Oliveira VZ. [Adherence to treatment in patients with cystic fibrosis]. J Bras Pneumol 2007;33(6):663-670. Article in Portuguese.

26. Myers LB, Horn SA. Adherence to chest physiotherapy in adults with cystic fibrosis. J Health Psychol 2006;11(6):915-926.

27. Arias Llorente RP, Bousoño García C, Díaz Martín JJ. Treatment compliance in children and adults with cystic fibrosis. J Cyst Fibros 2008;7(5):359-367.

28. Hobbs SA, Schweitzer JB, Cohen LL, Hayes AL, Schoell C, Crain BK. Maternal attributions related to compliance with cystic fibrosis treatment. J Clin Psychol Med Settings 2003;10(4):273-277.

29. Abbott J, Dodd M, Bilton D, Webb AK. Treatment compliance in adults with cystic fibrosis. Thorax 1994;49:(2):115-120.

30. White D, Stiller K, Haensel N. Adherence of adult cystic fibrosis patients with airway clearance and exercise. J Cyst Fibros 2007;6(3): 163-170.

31. White T, Miller J, Smith GL, McMahon WM. Adherence and psychopathology in children and adolescents with cystic fibrosis. Eur Child Adolesc Psychol 2009;18(2):96-104.

32. Daniels T, Goodacre L, Sutton C, Pollard K, Conway S, Peckham D. Accurate assessment of adherence: self-report and clinician report vs electronic monitoring of nebulizers. Chest 2011;140(2):425-432.

33. Latchford G, Duff A, Quinn J, Conway S, Conner M. Adherence to nebulised antibiotics in cystic fibrosis. Patient Educ Counsel 2009; 75(1):141-144.

34. McNamara PS, McCormack P, McDonald AJ, Heaf L, Southern $\mathrm{KW}$. Open adherence monitoring using routine data download from an adaptive aerosol delivery nebuliser in children with cystic fibrosis. J Cyst Fibros 2009;8(4):258-263.

35. Eakin MN, Bilderback A, Boyle MP, Mogayzel PJ, Riekert KA. Longitudinal association between medication adherence and lung health in people with cystic fibrosis. J Cyst Fibros 2011;10(4):258-264.

36. Burrows JA, Bunting JP, Masel PJ, Bell SC. Nebulised dornase alpha: adherence in adults with cystic fibrosis. J Cyst Fibros 2002; 1(4):255-259.

37. Adde FV, Rodrigues JC, Cardoso AL. Nutritional follow-up of cystic fibrosis patients: the role of nutrition education. J Pediatr 2004;80(6): 475-482.

38. Simon SL, Duncan CL, Horky SC, Nick TG, Castro MM, Riekert KA. Body satisfaction, nutritional adherence, and quality of life in youth with cystic fibrosis. Pediatr Pulmonol 2011;46(11):10851092.

39. Schall JI, Bentley T, Stallings VA. Meal patterns, dietary fat intake and pancreatic enzyme use in preadolescent children with cystic fibrosis. J Pediatr Gastroenterol Nutr 2006;43(5):651-659.

40. Gayer D, Ganong L. Family structure and mothers' caregiving of children with cystic fibrosis. J Fam Nurs 2006;12(4):390-412.

41. Borowitz D, Wegman T, Harris M. Preventive care for patients with chronic illness. Multivitamin use in patients with cystic fibrosis. Clin Pediatr 1994;33(12):720-725.

42. Hollander FM, de Roos NM, Dopheide J, Hoekstra T, van Berkhout FT. Self-reported use of vitamins and other nutritional supplements in adult patients with cystic fibrosis. Is daily practice in concordance with recommendations? Int J Vitam Nutr Res 2010; $80(6): 408-415$.

43. Anthony H, Paxton S, Bines J, Phelan P. Psychosocial predictors of adherence to nutritional recommendations and growth outcomes in children with cystic fibrosis. J Psychosom Res 1999; 47(6):623-634. 


\section{Adherence to Cystic Fibrosis Programs}

45. Lu M, Safren SA, Skolnik PR, Rogers WH, Coady W, Hardy H, Wilson IB. Optimal recall period and response task for self-reported HIV medication adherence. AIDS Behav 2008;12(1):86-94.

44. Walsh JC, Horne R, Dalton M, Burgess AP, Gazzard BG. Reasons for non-adherence to antiretroviral therapy: patients' perspectives provide evidence of multiple causes. AIDS Care 2001;13(6):709-720.

46. Woloshin S, Schwartz LM, Welch HG. Patients and medical statistics. Interest, confidence and ability. J Gen Intern Med 20(11):996-1000, 2005.

47. Quittner AL, Drotar D, Ievers-Landis CE, Seidner D, Slocum N, Jacobsen J. Adherence to medical treatments in adolescents with cystic fibrosis: the development and evaluation of family-based interventions. In: Drotar D, editor. Promoting adherence to medical treatment in childhood chronic illness: interventions and methods. Hillsdale, New Jersey: Lawrence Erlbaum Associates; 2000:383-407.

48. Quittner AL, Espelage DL, Ievers-Landis CE, Drotar D. Measuring adherence to medical treatments in childhood chronic illness: considering multiple methods and sources of information. J Clin Psychol Med Settings 2000;7(1):41-54.

49. Davies JC, Wainwright CE, Canny GJ, Chilvers MA, Howenstine MS, Munck A, et al. Efficacy and safety of ivacaftor in patients aged 6 to $11 \mathrm{y}$ with cystic fibrosis with a G551D mutation. Am J Respir Crit Care Med 2013;187(11):1219-1225.

50. Simmonds MJ, Olson SL, Jones S, Hussein T, Lee CE, Novy D, Radwan H. Psychometric characteristics and clinical usefulness of physical performance tests in patients with low back pain. Spine 1998;23(22):2412-2421.

51. Arnsten JH, Demas PA, Farzadegan H, Grant RW, Gourevitch MN, Chang CJ. Antiretroviral therapy adherence and viral suppression in HIV-infected drug users: comparison of self-report and electronic monitoring. Clin Infect Dis 2001;33(8):1417-1423.

52. Liu H, Golin CE, Miller LG, Hays RD, Beck CK, Sanandaji S, et al. A comparison study of multiple measures of adherence to HIV protease inhibitors. Ann Intern Med 2001;134(10):968-977.
53. Wagner GJ, Kanouse DE, Koegel P, and Sullivan G. Correlates of HIV antiretroviral adherence in persons with serious mental illness. AIDS Care 2004;16(4):501-506.

54. Rothert ML. Physicians' and patients' judgments of compliance with a hypertensive regimen. Med Decis Making 1982;2(2):179-195.

55. Bogart LM, Catz SL, Kelly JA, Benotsch EG. Factors influencing physicians' judgments of adherence and treatment decisions for patients with HIV disease. Med Decis Making 2001;21(1):2836.

56. Fullen BM, Doody C, Baxter GD, Daly LE, Hurley DA. Chronic low back pain: non-clinical factors impacting on management by Irish doctors. Ir J Med Sci. 2008;177(3):257-263.

57. Wagner GJ, Rabkin JG. Measuring medication adherence: are missed doses reported more accurately then perfect adherence? AIDS Care 2000;12(4):405-408

58. Barfod TS, Sørensen HT, Nielsen H, Rodkjaer L, Obel N. 'Simply forgot' is the most frequently stated reason for missed doses of HAART irrespective of degree of adherence. HIV Med 2006;7(5): 285-290.

59. Sabaté E. Defining adherence. Adherence to long-term therapies: evidence for action. World Health Organization, 2003. http:// www.who.int/chp/knowledge/publications/adherence_report/en. Accessed June 6, 2014.

60. Conway SP, Pond MN, Hamnett T, Watson A. Compliance with treatment in adult patients with cystic fibrosis. Thorax 1996;51(1): 29-33.

61. Logan AG. Transforming hypertension management using mobile health technology for telemonitoring and self-care support. Can J Cardiol 2013;29(5):579-585.

62. Ma J, Xiao L, Blonstein AC. Measurement of self-monitoring web technology acceptance and use in an e-health weight-loss trial. Telemed J E Health 2013;19(10):739-745.

63. El-Gayar O, Timsina P, Nawar N, Eid W. A systematic review of IT for diabetes self-management: are we there yet? Int J Med Inform 2013;82(8):637-652. 